\title{
Changes in Titin and Collagen Underlie Diastolic Stiffness Diversity of Cardiac Muscle
}

\author{
Yiming $\mathrm{Wu}^{1}$, Olivier Cazorla ${ }^{1}$, Dietmar Labeit ${ }^{2}$, Siegfried Labeit ${ }^{3}$ and \\ Henk Granzier ${ }^{1}$ \\ ${ }^{1}$ Department of VCAPP, Washington State University, Pullman, WA, 99164-6520, USA; \\ ${ }^{2}$ Department of Anesthesiology and Intensive Operative Medicine. University Hospital Mannheim, \\ 68135, Germany; ${ }^{3}$ EMBL, 69012 Heidelberg, Germany
}

\begin{abstract}
Y. Wu, O. Cazorla, D. Labeit, S. Labeit and H. Granzier. Changes in Titin and Collagen Underlie Diastolic Stiffness Diversity of Cardiac Muscle.

Small

(N2B) and large (N2BA) cardiac titin isoforms are differentially expressed in a species-specific and heart locationspecific manner. To understand how differential expression of titin isoforms may influence passive stiffness of cardiac muscle we investigated the mechanical properties of mouse left ventricular (MLV) wall muscle (expressing predominantly the small titin isoform), bovine left atrial (BLA) wall muscle (predominantly the large isoform), and bovine left ventricular (BLV) wall muscle (expressing small and large isoforms at similar levels). Results indicate that the overall passive muscle stiffness of the muscle types varies nearly ten-fold, with stiffness increasing in the following order: BLA, BLV and MLV. To investigate the basis of the variation in the overall muscle stiffness, the contributions of titin and collagen to muscle stiffness were determined. Results showed that increased muscle stiffness results from increases in both titin- and collagen-based passive stiffness, indicating that titin and collagen change in a co-ordinated fashion. The expression level of the small titin isoform correlates with titin's contribution to overall muscle stiffness, suggesting that differential expression of titin isoforms is an effective means to modulate the filling behavior of the heart.
\end{abstract}

Key Words: Connectin; Elasticity; Passive force; Diastole; Muscle mechanics.

\section{Introduction}

Titin is a giant protein that spans the half sarcomere of striated muscle and underlies the majority of the passive force of cardiac myocytes (for original citations see ${ }^{1}$ ). Titin's force arises from its I-band spanning region, which, in response to sarcomere stretch, extends and develops force. ${ }^{2,3}$ The extensible I-band region of cardiac titin has a complex sequence with three distinct extensible segments: (1) a PEVK segment, (2) serially linked immunoglobulin (Ig)-like domains that make up a proximal and a distal Ig segment, and (3) a unique sequence that is part of the N2B element. ${ }^{4}$ These three segments contribute to titin's extension along different parts of the physiological sarcomere-length (SL) range, and cardiac titin can be considered a three-element molecular spring. ${ }^{5,6}$

In the mammalian heart, two classes of titin isoforms exist that vary within their extensible Iband region: the so-called N2B isoform (containing the N2B sequence element) and N2BA isoform (containing both the N2B and N2A sequence elements). ${ }^{7}$ N2BA titin contains a much longer PEVK segment than N2B titin (600-800 $v 163$ residues) as well as additional Ig domains that make up a middle Ig segment. As a result of these sequence difference, N2BA titin has a higher molecular mass

Please address all correspondence to: Henk Granzier, Department of VCAPP, Washington State University, Pullman, WA, 99164-6520, USA. E-mail: granzier@wsunix.edu 
than N2B titin $(\sim 3.3 \vee 2.97 \mathrm{MDa}) .{ }^{7}$ Recent studies on myocardium isolated from various species have shown that the total amount of titin is constant ( 6 molecules per half thick filament) but that the expression level of $\mathrm{N} 2 \mathrm{~B}$ and $\mathrm{N} 2 \mathrm{BA}$ cardiac titin varies from predominantly $\mathrm{N} 2 \mathrm{~B}$ (rat and mouse) to predominantly N2BA (bovine atrium), with many species (including human) co-expressing the isoforms at similar levels. ${ }^{8}$

Although in recent years there has been considerable progress in understanding titin at levels that range from the single molecule ${ }^{9}$ to the single cardiac myocyte ${ }^{8}$ the relevance of titin to the overall behavior of muscle is not as well understood. Heart muscle also contains collagen, and the relative importance of collagen and titin to overall passive muscle force has been studied only in rat trabeculae, ${ }^{2}$ not in wall muscle or in species that express the N2BA isoform. To determine the relevance of differential expression of cardiac titin isoforms to the overall properties of the myocardium, we determined the contribution of titin and collagen to the overall stiffness of cardiac muscles that express predominantly N2B titin (mouse left ventricle), predominantly N2BA titin (bovine left atrium), or that co-express $\mathrm{N} 2 \mathrm{~B}$ and N2BA titins at similar levels (bovine left ventricle). The individual contributions of titin and collagen to diastolic stiffness were determined by using extraction and degradation techniques.

Results indicate that the maximal SL to which muscle can be reversibly stretched without a reduction in passive tension varies in the different muscle types. Near the maximal length of the reversible range, titin and collagen contribute about equally to passive tension, while at shorter SLs titin's contribution dominates. Thus, titin is the primary contributor to passive tension of cardiac muscle. Furthermore, titin-based and collagenbased stiffness both increase as the expression level of N2B titin goes up, indicating that the properties of titin and collagen change in a co-ordinated fashion and that they may play complementary roles in the filling behavior of the heart.

\section{Materials and Methods}

Muscle and myocytes

\section{Muscle}

Hearts were rapidly excised from 8- to 9-week-old mice (Balb/C, male). The left ventricle was opened immediately and placed in a modified Krebs solution [composition in mu: $\mathrm{NaCl} 122, \mathrm{KCl} 5, \mathrm{NaHCO}_{3}$ 25, $\mathrm{MgCl}_{2}$ 1.08, $\mathrm{NaH}_{2} \mathrm{PO}_{4}$ 1.3, $\mathrm{CaCl}_{2}$ 0.2, 2,3butanedione monoxime (BDM) 30, glucose 11.2, insulin $5 \mathrm{U} / \mathrm{l}$, gassed with $95 \% \mathrm{O}_{2}, 5 \% \mathrm{CO}_{2}$, pH 7.4]. Bovine hearts (animals 18 months old and weighing $\sim 1100 \mathrm{lbs}$ ) were obtained from local slaughterhouses. The hearts were excised typically within $\sim 20 \mathrm{~min}$ following death. The left atrium and left ventricle were cut into small pieces and placed into oxygenated Krebs solution. Small wall muscle strips (length: $1.5-3.0 \mathrm{~mm}$; diameter: $\sim 0.3 \mathrm{~mm}$ ) were dissected using a high-resolution binocular microscope. Extreme care was taken to dissect the strips in the direction of the fibers and to avoid stretching the preparations during dissection. Visual inspection under the dissection microscope revealed that some areas of the BLA contained high levels of collagen and these areas were avoided in this study. Muscle strips were skinned in relaxing solution containing $1 \% \mathrm{w} / \mathrm{v}$ Triton X-100 (for composition see ${ }^{8}$ ) overnight at $4^{\circ} \mathrm{C}$. The strips were then washed thoroughly with relaxing solution. To prevent degradation, all solutions contained protease inhibitors (PMSF: $0.5 \mathrm{~mm}$; leupeptin: $0.04 \mathrm{~mm}$ and E64: 0.01 mM).

\section{Myocytes}

Single myocytes were isolated from the mouse left ventricle, bovine left ventricle and bovine left atrium using the blending method explained in Cazorla et al. ${ }^{8}$ Briefly, small pieces $(\sim 1 \mathrm{~cm}$ long, $\sim 3 \mathrm{~mm}$ diameter) of myocardial tissue were pre-skinned by placing them for $50 \mathrm{~min}$ in relaxing solution containing $1 \%$ Triton $\mathrm{X}-100$ at $4^{\circ} \mathrm{C}$. The pieces were then placed in fresh relaxing solution and homogenized in a blender (Ultra-Turrax TP18 with a S25N-10G shaft, Ika Works, Inc., Wilmington, $\mathrm{NC}$, USA) for 6 to $10 \mathrm{~s} 12500 \mathrm{rpm}$, resulting in a suspension of small clumps of myocyte-sized preparations and cell fragments.

\section{Tension measurement}

\section{Muscle}

The set-up has been described previously. ${ }^{2}$ Briefly, passive tension was measured with a silicon semiconductor strain gauge (model AE-801, SensoNor, Horten, Norway). Muscle length was controlled by a high-speed motor (Model 308B, Aurora Scientific Inc., Richmond Hill, Ontario, Canada). Preparations were attached to the motor arm and the force transducer via aluminium clips. Using a small 
prism, the two perpendicular diameters of the muscle were measured and the cross-sectional area was calculated, assuming the cross-section is elliptical in shape. This area was used to calculate the force per unit area (in $\mathrm{mN} / \mathrm{mm}^{2}$ ), allowing us to compare results from different muscles. Stiffness was determined from the slope of the tension-SL relation. For calculating stiffness we normalized SL with respect to the slack SL, making the change in SL a unit-less value and the unit of stiffness $\mathrm{mN}$ / $\mathrm{mm}^{2}$. Experiments were performed at room temperature $\left(20-22^{\circ} \mathrm{C}\right)$.

\section{Myocytes}

Myocytes were added to a chamber mounted on the stage of a phase-contrast microscope. First, one end of a single cell was glued to a motor (Cambridge Technology, Watertown, MA, USA, type 308B). The free end was then positioned with a micromanipulator so that the myocyte axis aligned with the microscope optical axis, and the myocyte cross-section was imaged and its area measured (using image-processing techniques). The cross-sectional area of the myocyte was used to convert measured force to tension. Finally, the free end of the myocyte was glued to a force transducer (model 406A Aurora Scientific, Inc., resonant frequency $\sim 0.1 \mathrm{kHz}$ ). To minimize the disturbing effect of meniscus forces, the force transducer entered the chamber below the solution surface through the side of the chamber. A gravity-based perfusion system was used to replace solutions in the chamber. The cell was positioned at the tip of a manifold that received the outlet of eight micro-capillaries connecyted to 5-ml syringes filled with relaxing or activating solutions. Solutions were selected with pinch valves (ValveLink8, Automate Scientific, Inc., San Francisco, CA, USA) controlled by customwritten software. Experiments were performed at $20-22^{\circ} \mathrm{C}$.

\section{Sarcomere length (SL) measurement}

\section{Muscle}

SL was measured with laser-diffraction. The diffraction pattern was collected with a bright-field objective, a telescope lens was focused on the back focal plane of the objective, and the diffraction pattern was projected, after compression with a cylindrical lens, onto a photodiode array. The firstorder diffraction peak position was obtained using a digital spot-position detector board installed in an
IBM AT computer. This signal was converted to sarcomere length using a calibration curve that was established with diffraction peaks of a $25 \mu \mathrm{m}$ grating that was present in the chamber. For further details see ${ }^{2}$.

\section{Myocytes}

SL was measured on-line at a frequency of $60 \mathrm{~Hz}$, using a commercially available sarcomere length detection system (Ionoptix Corp., Milton, MA, USA). Measurements were based on Fourier analysis of optical density traces of the striation pattern obtained from digitized images of the cells. For additional details see Cazorla et al. ${ }^{8}$

\section{Protocols}

\section{Muscle}

Maximum active force was measured at a SL of $\sim 2.3 \mu \mathrm{m}$, using an activating solution with pCa 4.5 $\left(\mathrm{pCa}=-\log \left[\mathrm{Ca}^{2+}\right]\right)$. Passive force was measured while stretching the preparation with a constant velocity $(0.1$ length/s) from their slack length to a predetermined amplitude followed by a release to the slack length. Stretch-release cycles were followed by 15-min rest periods at the slack length. For thin and thick filament extraction the perfusate was switched sequentially to relaxing solution containing $0.6 \mathrm{M} \mathrm{KCl}(45 \mathrm{~min})$ and relaxing solution containing $1.0 \mathrm{M} \mathrm{KI}(45 \mathrm{~min})$. These solutions depolymerize the thick and thin filaments and thereby remove titin's anchors in the sarcomere. In other experiments, titin was degraded by treating muscle strips with $0.25 \mu \mathrm{g} / \mathrm{ml}$ trypsin (Sigma, St. Louis, MO, USA; 12400 BAEE units/mg) for varying times (cf. $\left.{ }^{10}\right)$.

\section{Myocytes}

The maximal active tension at SL $2.3 \mu \mathrm{m}$, and passive tension-SL relationship of each cell were measured using the same methods used for muscle. In addition to titin, intermediate filaments (IFs) also develop force in stretched myocytes. ${ }^{2}$ To determine the force produced by IFs, myocytes were incubated for $10 \mathrm{~min}$ with relaxing solution that contained $0.6 \mathrm{M} \mathrm{KCl}$ solution followed by a 30-min incubation in relaxing solution with $1.0 \mathrm{M} \mathrm{KI}$ (for details see ${ }^{2}$ ). The force-SL length relation following extraction was assumed to be IF-based. Considering the extraction-resistant nature of IFs, ${ }^{11}$ major changes in IFs are not expected, although minor effects of the 
$\mathrm{KCl} / \mathrm{KI}$ extraction protocol on IF-based tensions can not be excluded. IF-based forces were generally small ( $<5 \%$ of the total force). Experiment control and data collection were with a GP6-450 PC, a National Instruments data acquisition board (PCIMIO-16XE-10), and using custom-written routines in LabVIEW.

\section{Gel electrophoresis and Western blotting}

To determine the titin isoform composition, whether myosin and actin were well extracted $(\mathrm{KCl} / \mathrm{KI}$ experiments), and the degree of titin degradation (trypsin experiments), all preparations were solubilized and analyzed with 2-7\% SDS-PAGE, using a silver-staining method (cf. ${ }^{12}$ ). Western blotting with affinity-purified anti-titin antibodies specific to the $\mathrm{N} 2 \mathrm{~A}$ and $\mathrm{N} 2 \mathrm{~B}$ elements was performed as described in ${ }^{13}$. The locations of the peptides used to raise the antibodies in the titin sequence are shown in Figure 1(A).

\section{Statistics}

Results are shown as the mean \pm s.E., unless indicated otherwise. Significant differences were assigned to selected parameters using the Student's t-test or one way ANOVA and Tukey's multiple comparison with $P<0.05$.

\section{Results}

\section{Titin isoforms}

The SDS-PAGE result shown in Figure 1A reveals that the bovine left ventricle (BLV) contains a fastand a slow-migrating $\mathrm{T} 1$ band, the mouse left ventricle (MLV) a dominant fast-migrating T1 band, and bovine left atrium (BLA) a dominant slowmigrating band. Western blot results with N2B and N2A-specific anti-titin antibodies show that the fastmigrating $\mathrm{T} 1$ band reacts with anti-N2B antibodies only and the slow-migrating band with both antiN2B and anti-N2A antibodies (Fig. 1B-D). These findings indicate that mouse expresses predominantly N2B titin, cow atrium predominantly N2BA titin, and cow ventricle expresses high levels of both N2B and N2BA titin. These muscle specimens are thus well-suited for investigating the relation between titin isoform expression and passive muscle stiffness.
Passive properties of cardiac muscle

To determine the maximum SL that can be repeatedly reached without reducing passive tension, the skinned muscles were stretch-released in relaxing solution with progressively increasing amplitudes, with a 15-min rest period at the slack length in between the stretch-release cycles. The passive tension-SL curve was reproducible at SLs below $\sim 2.3 \mu \mathrm{m}$ in MLV and below $\sim 2.5 \mu \mathrm{m}$ in both BLA and BLV (data not shown). Reaching longer SLs resulted in a permanent reduction of passive tension. It is likely that this results from damage to collagen, because isolated cardiac myocytes can be stretched to a SL of at least $2.7 \mu \mathrm{m}$ without inflicting permanent damage. ${ }^{2}$ All reported results on cardiac muscle were obtained within their damage-free SL range.

The muscles were studied before and after extracting the preparations with $\mathrm{KCl}$ and $\mathrm{KI}$. The total passive tension before and after extraction differed greatly in the different muscle types, with tension being highest in MLV, lowest in BLA and intermediate in BLV. Figure 2 shows typical examples; the mean results are shown in Figure 3(A). Stiffness-SL data (stiffness: slope of the tension-SL relation) are shown in the inset of Figure 3(A). Total stiffness of the mouse myocardium is significantly higher than that of bovine myocardium, with bovine ventricle being stiffer than bovine atrium.

SDS-PAGE of MLV, BLA and BLV muscle revealed that the $\mathrm{KCl} / \mathrm{KI}$ treatment removed $>95 \%$ of myosin and actin (results not shown). Thus, this treatment removes titin's anchors in the sarcomere, abolishing titin as a contributor to passive tension. To test whether $\mathrm{KCl} / \mathrm{KI}$ extraction also affects the passive tension developed by collagen, we dissected small collagen strips from cow left atrium and left ventricle (identity of the strips based on their white color and absence of response to activating solution) and measured their force-extension curve before and after exposure to $\mathrm{KCl} / \mathrm{KI}$ (treatment identical to that of muscle strips). The stretch amplitude was adjusted so that the peak tension generated before extraction was comparable to that of the muscle preparations. The peak passive tension decreased from $35.9 \pm 2.1 \mathrm{mN} / \mathrm{mm}^{2}(n=5)$ to $35.3 \pm 2.1 \mathrm{mN} /$ $\mathrm{mm}^{2}$, or $1.6 \%$. This decrease is very small and similar in size to 'run-down' of repeatedly stretchreleased collagen strips. Thus it appears that the $\mathrm{KCl} / \mathrm{KI}$ treatment has no major effect on cardiac collagen.

To study further whether the $\mathrm{KCl} / \mathrm{KI}$ extraction method specifically abolishes the titin-based passive tension of muscle, we compared this method with 


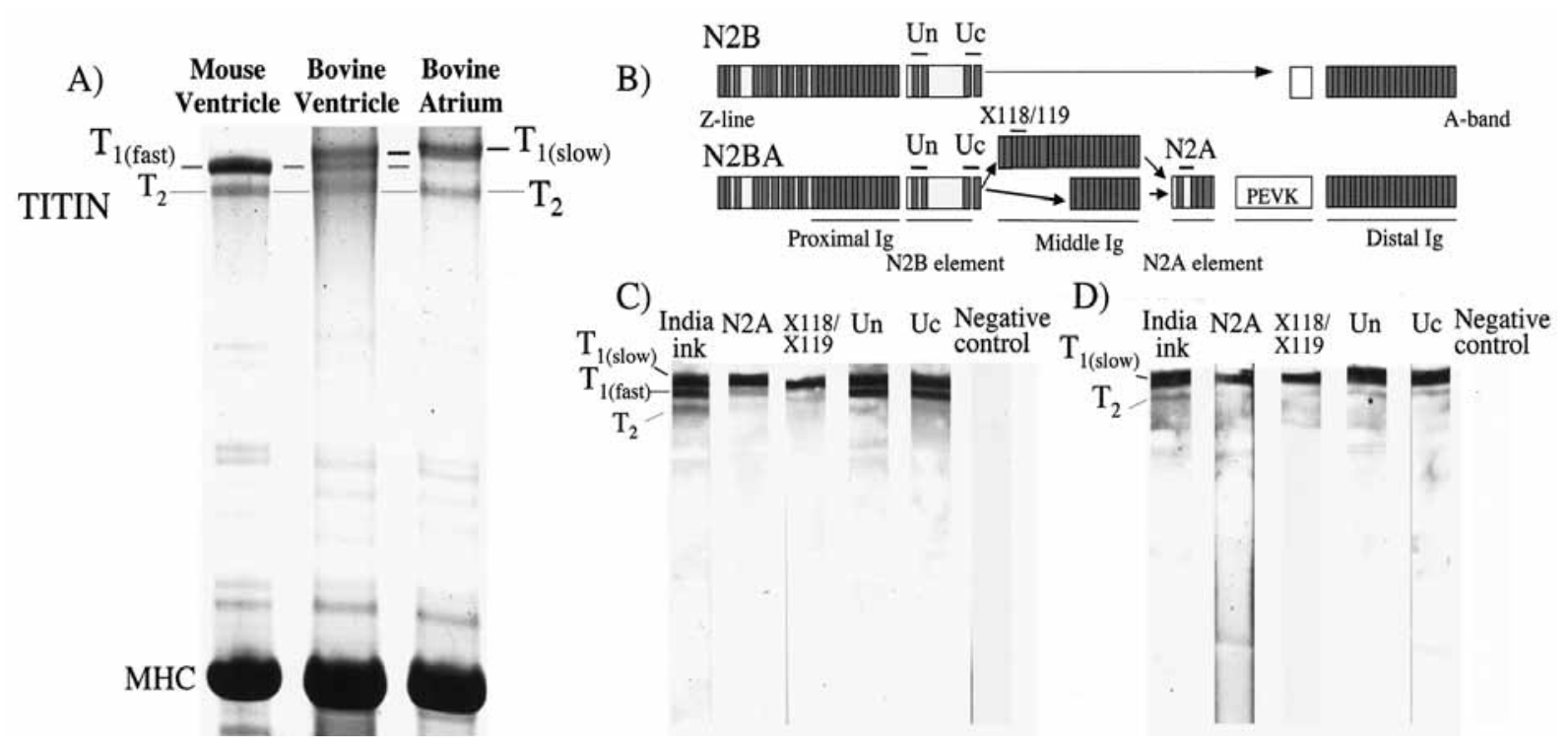

Figure 1 (A) SDS-PAGE of mouse left ventricle (MLV), bovine left ventricle (BLV) and bovine left atrium (BLA). MLV contains a fast-migrating $\mathrm{T}_{1}$ band $\left(\mathrm{T}_{1 \text { (fast) }}\right)$, and BLA a slow-migrating $\mathrm{T}_{1}$ band $\left(\mathrm{T}_{1 \text { (slow) }}\right)$. BLV contains both fast- and slow-migrating $\mathrm{T}_{1}$ bands. ( $\mathrm{T}_{2}$ is a degradation product of $\mathrm{T}_{1}$; for details see ${ }^{13}$ ). (B) Sequence of I-band region of $\mathrm{N} 2 \mathrm{~B}$ and N2BA cardiac titins (from ${ }^{7}$ ). N2BA titins contain a middle Ig segment with a varying number of Ig domains ${ }^{7}$ and the isoforms with the lowest (12) an highest (25) number of domains are shown. Also indicated are the locations of the antigens in titin's sequence that were used to raise anti-N2B and anti-N2A antibodies (see short horizontal bars). These antibodies allow us to distinguish N2B and N2BA titins (for additional information see ${ }^{7}$ ). (Dark gray blocks: Ig domains; light gray: unique sequences; white: PEVK). (C) and (D) Western blots of BLV (C), and BLA (D) titin with isoform-specific anti-titin antibodies. $\mathrm{T}_{1 \text { (fast) }}$ reacts only with anti-N2B antibodies while $\mathrm{T}_{1 \text { (slow) }}$ reacts with both anti-N2B and anti-N2A antibodies. This suggests that $\mathrm{T}_{1 \text { (rast) }}$ represents a N2B isoform and $\mathrm{T}_{1 \text { (slow) }}$ a N2BA titin isoform. (Negative control: first antibody omitted.)

the trypsin-based titin degradation method. ${ }^{2}$ Figure 4(A) inset (lane 2) shows that trypsin can be used to degrade titin without major effects on other proteins. (This conclusion agrees with several biochemical and functional studies. ${ }^{2}$ ) When mouse muscle was treated with trypsin, passive tension was clearly reduced [Fig. 4(A)], curve marked "trypsin treated"). Trypsin-treated muscles were then extracted witk $\mathrm{KCl} / \mathrm{KI}$. No additional reduction in passive tension is expected if the $\mathrm{KCl} / \mathrm{KI}$ method is specific for titin, because titin was already degraded. Indeed, the treatment had no significant additional effect [Fig. 4(A)], curve 3 marked "trypsin-treated and KCl.KI extracted"). (Note that when the order was reversed, i.e. first a $\mathrm{KCl} / \mathrm{KI}$ extraction and then a trypsin treatment, $\mathrm{KCl} / \mathrm{KI}$ extraction maximally depressed passive tension without an additional effect of trypsin, suggesting that both methods are specific for titin.)

We also compared the titin-based passive tension-SL relations of muscle with those of myocytes, using the $\mathrm{KCl} / \mathrm{KI}$ extraction method. Results revealed that in both mouse and bovine $\mathrm{LV}$, passive tension of muscle and cells varied only to a minor degree [Fig. 4(B), top and middle panels] while in bovine LA they were indistinguishable [Fig. 4(B), bottom]. Considering how different the two types of preparations are, the similarity in results of muscle and cells is remarkable. In sum, it seems justified to assume that the $\mathrm{KCl} / \mathrm{KI}$-sensitive tension of cardiac muscle is titin-based tension and that the $\mathrm{KCl} / \mathrm{KI}$-insensitive tension is collagen-based tension.

Both titin-based passive tension [Fig. (3B)] and collagen-based tension [Fig. 3(C)] vary significantly between the different muscle types, with the highest tensions in MLV, the lowest in BLA and intermediate tensions in BLV. The insets of Figures 3(B) and 3(C) show the titin-based [3(B)] and collagen-based [3(C)] passive stiffness. Results reveal that both titin- and collagen-based stiffness decrease in the following order: MLV, BLV, and BLA. Titin- and collagen-based stiffness are shown superimposed in Figure 3(D). In both BLV and BLA, titin-based stiffness is higher than collagen-based stiffness along the full SL-range studied. In MLV, titin-based stiffness is higher than collagen-based stiffness at SLs $<\sim 2.15 \mu \mathrm{m}$, but lower at SLs $>\sim 2.15 \mu \mathrm{m}$.

Titin-based tension is not proportional to the total cross-sectional area of the specimen, but instead to its myofibrillar cross-sectional area. To assess indirectly the fractional area of myofibrils in different preparations we measured their maximal 


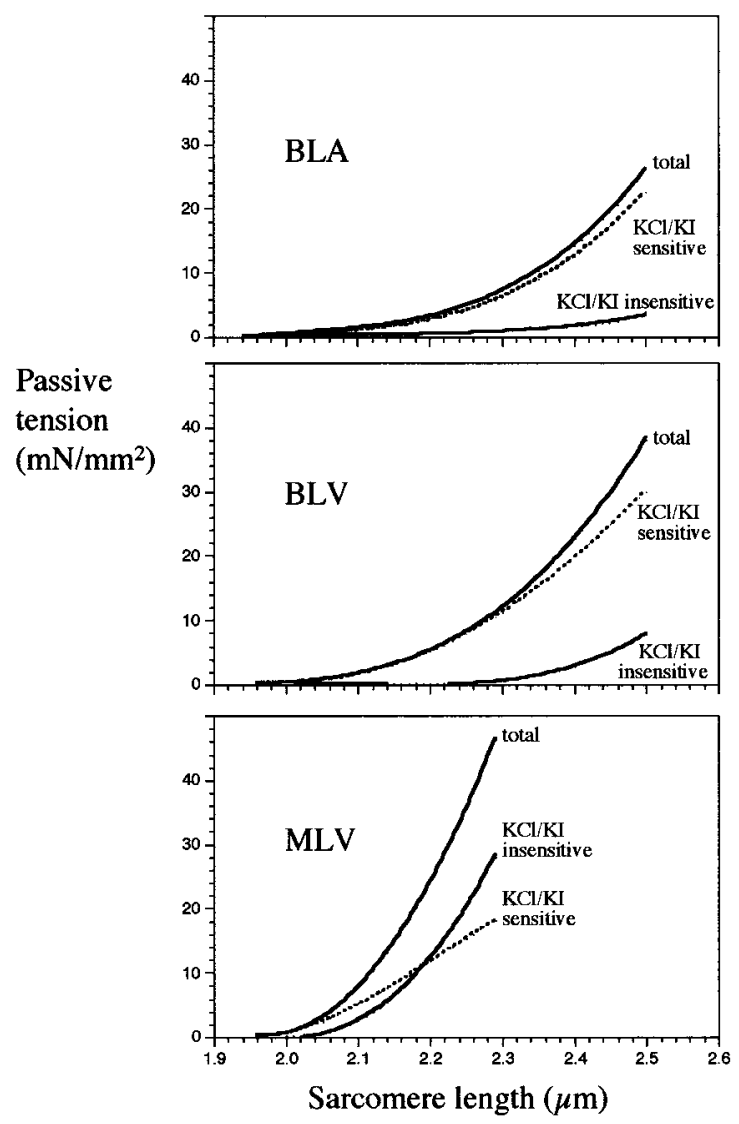

Figure 2 Typical examples of passive tension-SL relations of MLV, BLV, and BLA muscles. Tension before $\mathrm{KCl} / \mathrm{KI}$ extraction (curve marked "total") and tension following extraction (curve marked " $\mathrm{KCl} / \mathrm{KI}$ insensitive tension") were measured. $\mathrm{KCl} / \mathrm{KI}$ sensitive tension was obtained by subtracting the latter from the former. The tensions of the different muscle types vary greatly.

active tensions. The maximal active tension is proportional to the myofibrillar fractional area and is independent of the expression pattern of isoforms of contractile and/or regulatory proteins. ${ }^{14-16}$ Table 1 shows the maximal active tension in cardiac muscle and isolated myocytes. Multiple comparisons indicate that there are no significant differences between the active tensions developed by the different types of preparations. Thus, the similar maximal active tensions of the different muscle types suggest that their myofibrillar fractional areas are similar as well. This conclusion agrees with a recent ultrastructural study on mouse and pig cardiac myocytes. ${ }^{8}$

To determine the relative contribution of titin and collagen to overall passive tension of muscle, we determined titin- and collagen-based tensions as fractions of the total muscle tension. The data shown in Figure 3 were used for this purpose, as well as those from follow-up experiments on bovine left atrium in which the SL range was increased to probe more of the high-tension region of collagen (while just staying within the reversible tension range). In the mouse, titin develops $>80 \%$ of the total tension at SLs up to $\sim 2.1 \mu \mathrm{m}$, while in bovine LA and LV titin contributes $>80 \%$ at SLs up to $\sim 2.4 \mu \mathrm{m}$. At longer SLs, titin's contribution decreases and the contribution of collagen increases (see Fig. 5).

\section{Discussion}

Diastolic properties of cardiac muscle influence the filling behavior of the heart including the maximum diastolic volume, a volume that sets the systolic performance via the Frank-Starling relation. Here we studied the contribution of titin and collagen to the diastolic properties of cardiac muscle types that express different titin isoforms. In all investigated muscle types, titin is a primary contributor to passive muscle stiffness. Titin-based stiffness is highest in muscle expressing predominantly N2B titin, lowest in muscle expressing predominantly N2BA titin, and intermediate in muscle expressing both $\mathrm{N} 2 \mathrm{~B}$ and N2BA titins. Furthermore, collagen-based stiffness correlates with titin-based stiffness, and their co-ordinated change is responsible for the large variation in passive muscle stiffness.

\section{Methods to study the contribution of titin and collagen to muscle stiffness}

Titin is a long, slender molecule that is anchored in and near the Z-line as well as along the thick filament. ${ }^{17}$ Due to these multiple anchoring sites, methods to extract specifically titin from the sarcomere are currently unavailable. The $\mathrm{KCl} / \mathrm{KI}$ method used in this work is an indirect method and consists of extracting skinned muscle with $0.6 \mathrm{M} \mathrm{KCl}$ to remove the thick filaments and $1.0 \mathrm{M}$ KI to remove the thin filaments. ${ }^{2,18}$ This treatment removes titin's anchors in the sarcomere and, thus, renders titin ineffective as a source of passive force. The $\mathrm{KCl} / \mathrm{KI}$ extraction method has been used previously on single cardiac myocytes and skinned skeletal muscle fibers, preparations that do not contain collagen as a contributor to passive tension. To determine the suitability of $\mathrm{KCl} / \mathrm{KI}$ extraction for studying titin's contribution to passive tension of cardiac muscle (where collagen is present), we studied whether this extraction method affects collagen. Results indicate that $\mathrm{KCl} / \mathrm{KI}$ extraction does not affect collagen-based passive tension of either 

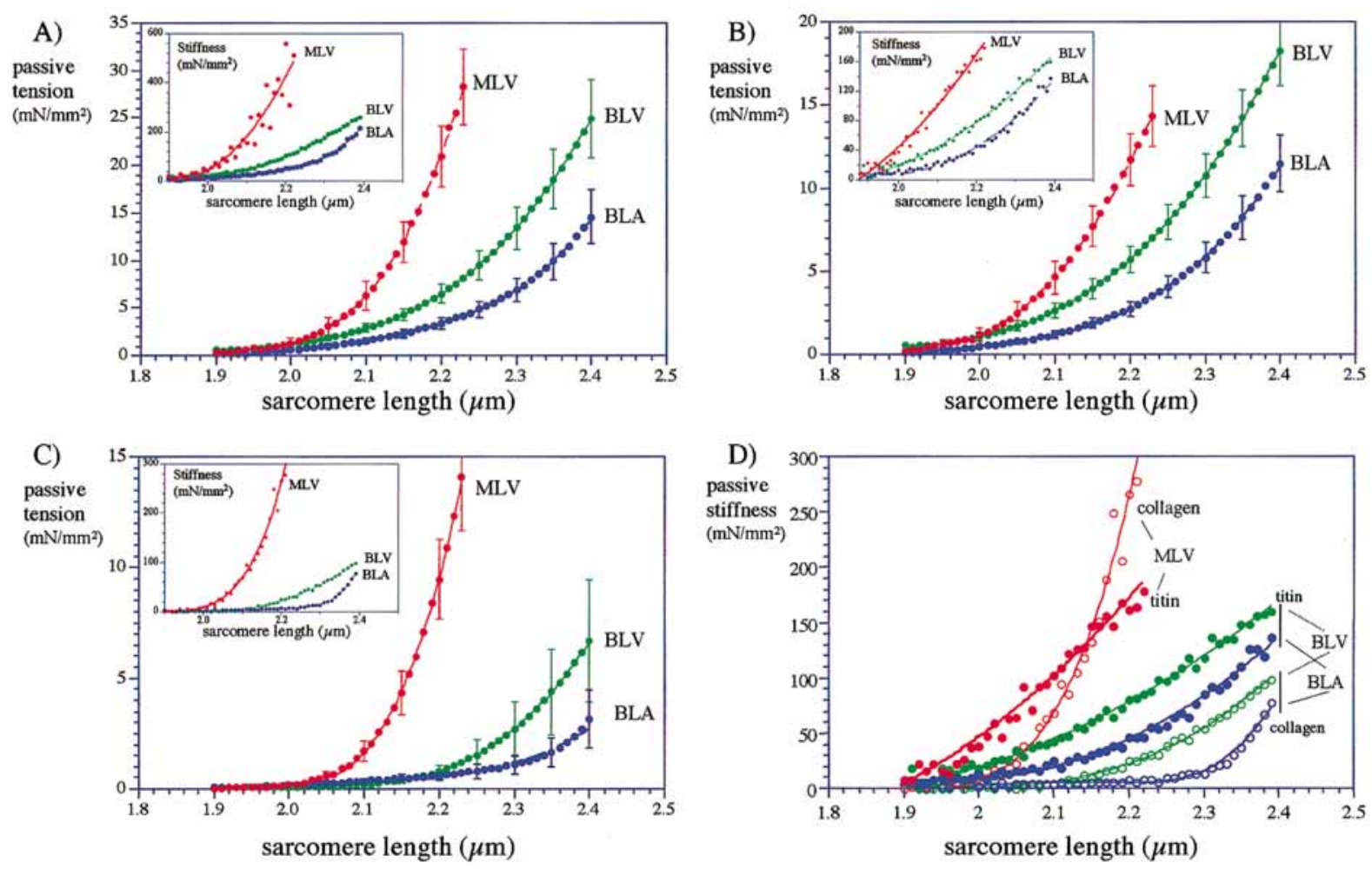

Figure 3 Average passive tension-SL relation of MLV, BLV and BLA muscles (mean \pm S.D.). (A) Total passive tension-SL relations. (B) Titin-based passive tension-SL relations. (Titin-based tension derived from the $\mathrm{KCl} / \mathrm{KI}$ sensitive tension.) (C) Collagen-based passive tension-SL relations. (Collagen-based passive tension was derived from the $\mathrm{KCl} / \mathrm{KI}$ insensitive tension.) The insets of A-C show the passive stiffness-SL relations. (D) Comparison of titin-based and collagen-based passive stiffness of MLV, BLV and BLA. (Ten muscle strips were used in each group.)

cardiac muscles or pure collagen strips. This finding is consistent with the finding of Medugorac and $\mathrm{Jacob}^{19}$ that collagen of cardiac muscle is highly resistant to salt extraction. To explore further whether $\mathrm{KCl} / \mathrm{KI}$ treatment of cardiac muscle only affects titin and not collagen, we compared the effect of $\mathrm{KCl} / \mathrm{KI}$ treatment of cardiac with that of trypsin. Trypsin is a proteolytic enzyme that splits peptide bonds on the carboxyl side of lysine and arginine. Trypsin has been used previously on cardiac myocytes, where it degrades the extensible region of titin. ${ }^{10}$ Within the extensible region, the PEVK segment appears most prone to degradation, ${ }^{10}$ probably as a result of its disordered conformation (which promotes accessibility for trypsin), and its high lysine content. ${ }^{4}$ Due to titin's high sensitivity for trypsin, conditions can be optimized such that titin is the main protein that is degraded. ${ }^{2}$ Our finding that the decrease in passive tension following trypsin treatment is similar in magnitude to that seen following $\mathrm{KCl} / \mathrm{KI}$ extraction supports the idea that $\mathrm{KCl} / \mathrm{KI}$ abolishes only titin's passive tension and not the collagen-based tension. This conclusion is further supported by the similarity in
$\mathrm{KCl} / \mathrm{KI}$-sensitive tension obtained in cardiac muscle and single cardiac myocytes [Fig. 3(B)].

\section{Contribution of collagen to passive stiffness}

Collagen is an extra-cellular matrix component that contributes to passive tension in stretched muscle, thereby influencing the end-diastolic volume of the heart. We found that collagen-based tension is similar following titin removal via either $\mathrm{KCl} / \mathrm{KI}$ extraction or trypsin-based digestion. This suggests that the reduction in lateral stiffness that results from the $\mathrm{KCl} / \mathrm{KI}$-based extraction of myofilaments does not significantly affect the collagen-based tension. The collagen-based tension-SL relation of the mouse $\mathrm{LV}$ muscle increases steeply at a SL of $\sim 2.3 \mu \mathrm{m}$, a finding that is similar to that reported earllier for the rat. $^{2}$ The steep increase in passive tension at a SL of $\sim 2.3 \mu \mathrm{m}$ is consistent with the morphological study by Hanley et al. ${ }^{20}$ that revealed that the perimysial collagen fibers of rat myocardium are wavy at short SLs and become straight at a SL of $\sim 2.3 \mu \mathrm{m}$. Thus, it is likely that in rat and mouse muscle, collagen is 

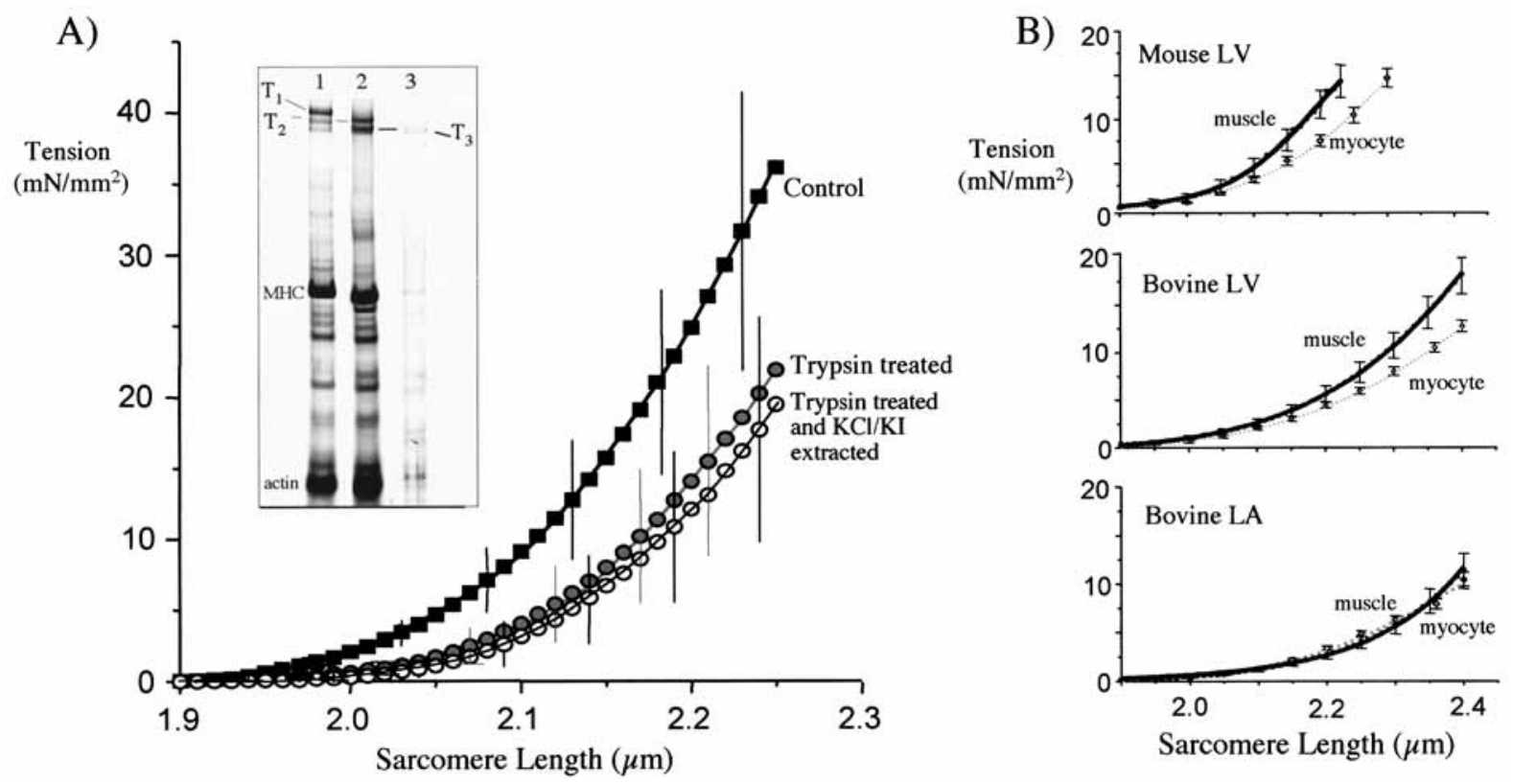

Figure 4 (A) Total passive tension (filled squares), tension after a 35-min trypsin treatment (closed circles), and tension after a 35-min trypsin treatment plus a subsequent $\mathrm{KCl} / \mathrm{KI}$ treatment (open circles) of mouse left ventricular muscle. (Mean \pm s.D. of four preparations.) The trypsin treatment greatly decreased passive tension with only a minimal additional reduction following the subsequent $\mathrm{KCl} / \mathrm{KI}$ extraction. Inset: SDS-PAGE of mouse muscle. Lane 1, control; lane 2, trypsin-treated for $35 \mathrm{~min}$; lane 3, $\mathrm{KCl} / \mathrm{KI}$-treated. The trypsin treatment resulted in the complete degradation of $\mathrm{T}_{1}$ (full-length molecule) into the degradation products $\mathrm{T}_{2}$ and $\mathrm{T}_{3}\left(\mathrm{see}^{10}\right)$. The $\mathrm{KCl} / \mathrm{KI}$ treatment removed nearly all myosin heavy chain (MHC) and actin, as well as their associated proteins (including titin). (B) KCl/KI-sensitive tension of muscle (dark lines) and single cardiac myocytes (light lines). Differences between muscle- and myocyte-derived tensions are insignificant. (Ten muscle strips and 12 myocytes were used in each group.)

Table 1 Cross-sectional area and maximum active tension

\begin{tabular}{|c|c|c|c|c|}
\hline & $\begin{array}{l}\text { Cross-sectional area } \\
\text { of muscle }\left(\mathrm{mm}^{2}\right)\end{array}$ & $\begin{array}{c}\text { Active tension } \\
\text { of muscle }\left(\mathrm{mN} / \mathrm{mm}^{2}\right)\end{array}$ & $\begin{array}{l}\text { Cross-sectional area } \\
\text { of myocyte }\left(\mu \mathrm{m}^{2}\right)\end{array}$ & $\begin{array}{c}\text { Active tension } \\
\text { of myocyte }\left(\mathrm{mN} / \mathrm{mm}^{2}\right)\end{array}$ \\
\hline MLV & $\begin{array}{l}0.12 \pm 0.02 \\
\quad(n=10)\end{array}$ & $\begin{array}{l}37.0 \pm 1.7 \\
\quad(n=10)\end{array}$ & $\begin{array}{r}309 \pm 39 \\
(n=11)\end{array}$ & $\begin{array}{l}31.3 \pm 3.8 \\
(n=10)\end{array}$ \\
\hline BLA & $\begin{array}{c}0.13 \pm 0.01 \\
(n=10)\end{array}$ & $\begin{array}{c}32.9 \pm 1.8 \\
(n=10)\end{array}$ & $\begin{array}{r}261 \pm 27 \\
(n=12)\end{array}$ & $\begin{array}{c}31.3 \pm 5.6 \\
(n=9)\end{array}$ \\
\hline BLV & $\begin{array}{c}0.12 \pm 0.02 \\
(n=10)\end{array}$ & $\begin{array}{l}38.8 \pm 1.9 \\
(n=10)\end{array}$ & $\begin{array}{r}303 \pm 31 \\
(n=12)\end{array}$ & $\begin{array}{l}31.4 \pm 5.3 \\
\quad(n=8)\end{array}$ \\
\hline
\end{tabular}

Values are mean \pm s.E.M.

responsible for limiting extension of the myocardium to a SL of $\sim 2.3 \mu \mathrm{m}$.

In the cow, collagen-based myocardial stiffness is less than that in the mouse, and a longer maximal diastolic SL can be reached without inflicting permanent mechanical damage to collagen $(\sim 2.5 \mu$ m in cow $v \sim 2.3 \mu \mathrm{m}$ in mouse). These mechanical differences in collagen are consistent with the reported type I/type III collagen ratio of 2.1 in cow $v 3.6$ in rat. ${ }^{19}$ Type I collagen forms large, well-structured fibers that resist extension, while type III forms a fine reticular network that is more compliant. ${ }^{19}$ Thus, the low type I/type III ratio of the cow may underlie the low collagen-based myocardial stiffness of this species.
The variation in diastolic stiffness of cardiac muscles results from changes in collagen- and titinbased stiffness [Fig. 3(A-C)]. Considering the "steplike" tension-SL curve of collagen (low tension at short to intermediate SLs followed by a steep increase in tension at long SLs), collagen may be most important in limiting the end-diastolic volume. The low collagen-based stiffness of the cow suggests that longer end-diastolic SLs may be reached in the cow than that in the mouse, but whether this is indeed the case remains to be investigated. Titinbased stiffness, on the other hand, increases more gradually with SL and contributes to diastolic stiffness at short-to-intermediate SLs, where collagen 


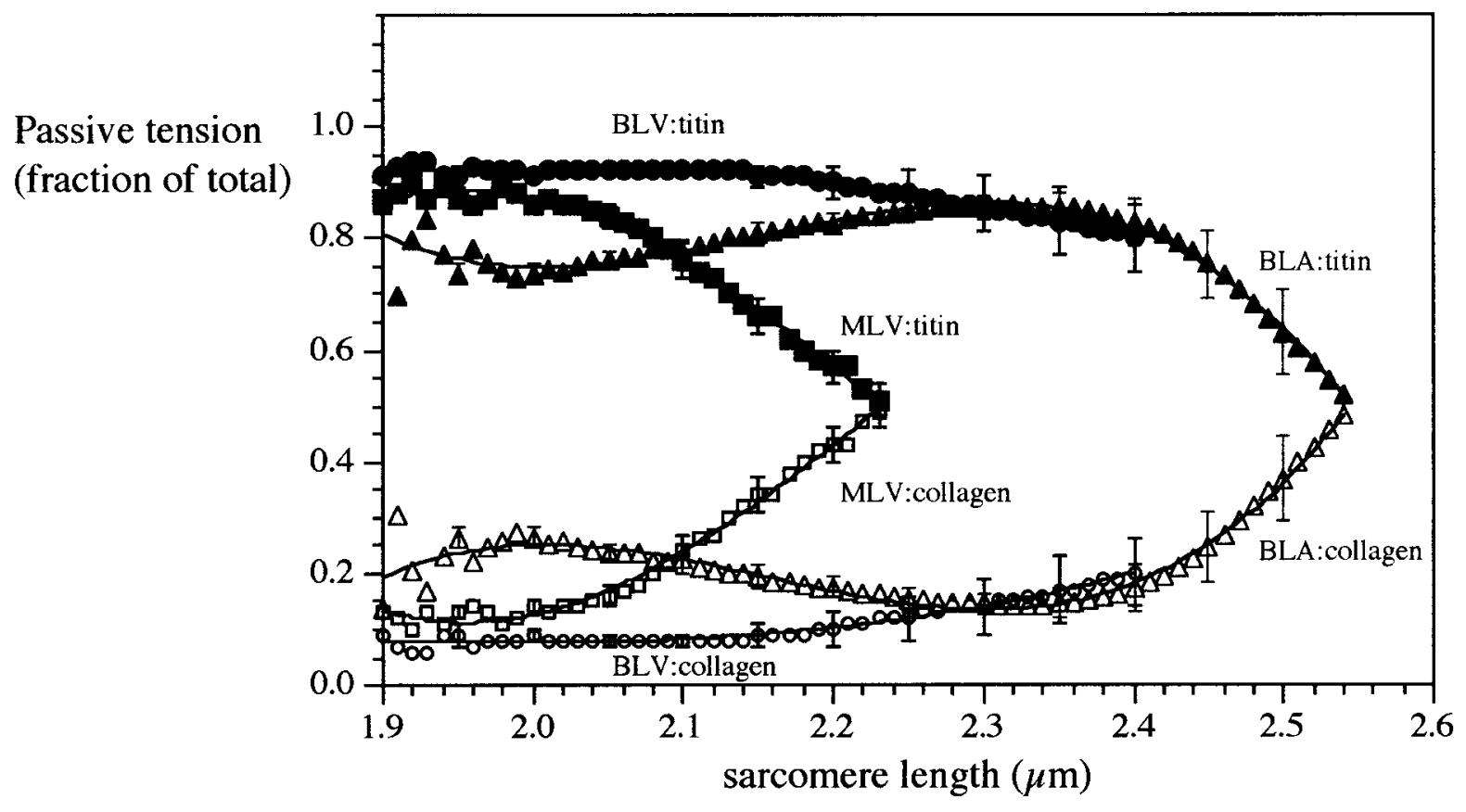

Figure 5 SL dependence of contribution of titin (closed symbols) and collagen (open symbols) to total passive tension. In bovine atrial (triangles) and bovine ventricular muscle (circles), titin contributes $>\sim 80 \%$ to total tension at SLs $<\sim 2.4 \mu \mathrm{m}$ while in mouse muscle (squares) titin contributes $<80 \%$ at SLs $<2.1 \mu \mathrm{m}$. At longer SLs, titin's contribution decreases and collagen becomes more important.

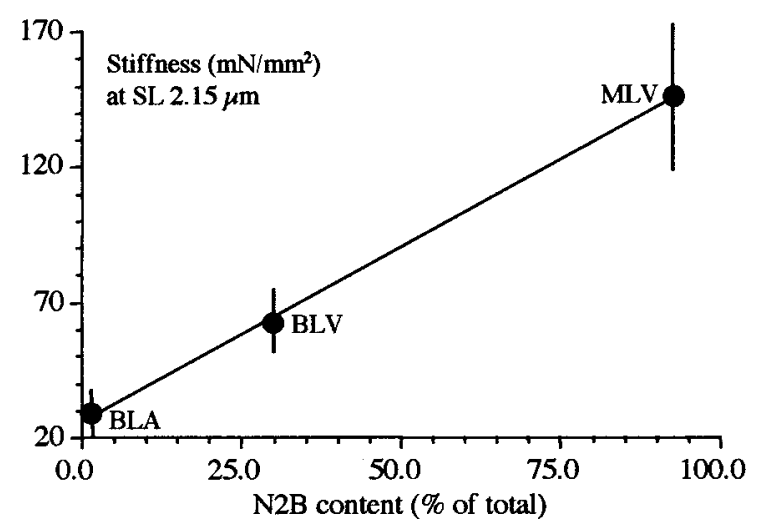

Figure 6 Relation between titin-based stiffness (from Fig. 3) and the N2B content (from ${ }^{8}$ ) of BLA, BLV and MLV.

contributes little. Thus, it is likely that collagen and titin are both important in determining the diastolic stiffness of myocardium and that they play complementary roles, explaining why, in tissues with different overall passive muscle stiffness, collagen and titin vary in a co-ordinated fashion.

\section{Variation in titin-based stiffness}

Our work revealed that titin-based passive stiffness is highest in mouse $\mathrm{LV}$, lowest in cow LA and intermediate in cow $\mathrm{LV}$, tissues that express predominantly N2B, predominantly N2BA, and both N2B and N2BA titins, respectively. Can these stiffness differences be explained by the differences in titin isoform composition? To answer this requires inspection of the sequence of titin [Fig. 1(B)]. In addition to the sequences that make up the extensible region of $\mathrm{N} 2 \mathrm{~B}$ titin, the extensible region of the N2BA isoform also contains the N2A splice element, a middle Ig segment and 400-600 additional PEVK residues. ${ }^{7}$ As a result, the contour length (end-to-end length when completely 
straight) of the extensible I-band region of N2BA titin is much longer than that of N2B titin. Thus, at a given SL the fractional extension (end-to-end length divided by the contour length) of titin's extensible region is much lower in N2BA than in N2B titin. Considering that titin's force is likely to be entropic in nature, and that entropic force increases with the segment's fractional extension $\left(\right.$ see $\left.^{21}\right)$, it follows from the lower predicted fractional extension of N2BA titin that passive force is lower for N2BA than N2B titin. Thus, the high titin-based passive stiffness of mouse $\mathrm{LV}$ and the low stiffness of cow LA are consistent with their high N2B and high N2BA content, respectively. Furthermore, the finding that the stiffness of bovine $\mathrm{LV}$ is intermediate between that of mouse LV and bovine LA is consistent with its co-expression of N2B and N2BA isoforms. The relation between the contribution of titin to muscle stiffness and the level of N2B expressed by the investigated muscle types is graphically depicted in Figure 6. A strong correlation $\left(r^{2}\right.$ of linear fit: 0.999) is apparent. It may be concluded that differential expression of titin isoforms is an effective means for modulating myocardial passive stiffness.

\section{Physiological significance of stiffness variation}

Titin performs a range of functions, including giving rise to a significant part of the passive tension of the myocardium (Fig. 5), maintaining sarcomeric integrity during contraction, and functioning as a scaffold in myofibrillogenesis. (For original citations see ${ }^{1}$ ). Any of these functions may be altered as a result of differential expression of titin isoforms. Titin's contribution to restoring force is worthwhile focusing upon. The extensible region of titin functions as a bi-directional molecular spring ${ }^{10}$ that in sarcomeres shortened to below the slack length $(\sim 1.9 \mu \mathrm{m})$ gives rise to restoring force (i.e., a force that pushes the Z-lines away from each other) and in sarcomeres stretched above the slack length to passive force (i.e., a force that pulls the Z-lines toward each other). Restoring and passive forces are symmetrical with respect to the slack length, i.e., for a given degree of shortening below the slack length, the restoring force is similar in magnitude to the passive force developed for the same degree of stretch above the slack length. ${ }^{10}$ Thus, although restoring forces were not studied in the present work, the measured passive forces indicate that the restoring forces should be highest in N2B-expressing myocardium (mouse and rat), and lowest in N2BAexpressing myocardium (bovine atrium).
Whether the in vivo end-systolic SL is below the slack length $(\sim 1.9 \mu \mathrm{m})$, a requirement for titin to develop restoring force, is not well established. Rodriguez et al. ${ }^{22}$ reconstructed myocardial SLs in isolated beating canine hearts and found that as the ventricular chamber volume was varied over its normal range of operations, the range of SLs is between $\sim 1.7$ and $\sim 2.4 \mu \mathrm{m}$. Thus, it may be possible that the end-systolic SL of large mammals is short enough for titin to give rise to restoring forces, especially under conditions where contractility is high and endsystolic volumes are small, such as during intense physical activity. That the same may be true for small rodents is suggested by their large (30-50\%) shortening fraction (SF), ${ }^{23,24}$ their relatively short end-diastolic SL $(\sim 2.2 \mu \mathrm{m}),{ }^{25,26}$ and the assumption that mid-wall SL changes in proportion to the SF. Hence, we hypothesize that in vivo, titin develops restoring force and that high restoring forces in small mammals are required to achieve more rapid early diastolic filling (diastole in mouse lasts $\sim 50 \mathrm{~ms}$ and in cow $\sim 500 \mathrm{~ms}$ ). To test this proposal will require detailed study of the in vivo diastolic SL range in small and large mammals.

Above the slack SL, titin's passive force is expected to play a role in determining the end-diastolic volume, and the high titin-based passive force provided by N2B titin may allow rapid and stable determination of this volume at the high beat frequencies encountered in small mammals. Above the slack SL, titin is also expected to play a role in centering the A-band within the sarcomere. ${ }^{1}$ The Aband may move off-center (shift) during systole, as a result of, for example, micro-variation in the calcium concentration in different parts of the sarcomere. During diastole, titin-based passive force will reset the A-band to its central location in the sarcomere, avoiding a progressive shift of the A-band with each systole. The rapid and frequent activation in small rodents is expected to result in a more pronounced A-band shift, and the high N2B-based passive force may function to reset the A-band rapidly to a central location during each diastole.

In summary, titin develops restoring as well as passive forces and we propose that the different levels of force that result from differential expression of titin isoforms allow for tuning of the heart's filling behavior in animals with different beat frequencies.

\section{Acknowledgements}

We greatly appreciate Mark MacNabb for technical support. This work was supported by La 668/6-1 (to SL), and NIH (HL61497 and HL62881) (to HG). 


\section{References}

1. Gregorio C, Granzier H, Sorimachi H, Labeit S. Muscle assembly: a titanic achievement? Curr Opin Cell Biol 1999; 11: 18-25.

2. Granzier H, Irving T. Passive tension in cardiac muscle: contribution of collagen, titin, microtubules, an intermediate filaments. Biophys J 1995; 68: 1027-1044.

3. Trombitas K, Jin JP, Granzier H. The mechanically active domain of titin in cardiac muscle. Circ Res 1995; 77: 856-861.

4. LABeit S, KOLMERER B. Titins: giant proteins in charge of muscle ultrastructure and elasticity. Science 1995; 270: 293-296.

5. Trombitas K, Freiburg A, Centner T, Labeit S, GranzIER H. Molecular dissection of N2B cardiac titin's extensibly. Biophys J 1999; 77: 3189-3196.

6. Linke W, Rudy D, Centner T, Gautel M, Witt C, LABEIT S, GREgorio C. I-band titin in cardiac muscle is a three-element molecular spring and is critical for maintaining thin filament structure. J Cell Biol 1999; 146: 631-644.

7. Freiburg A, Trombitas K, Hell W, Cazorla O, Fougerousse F, Centner T, Kolmerer B, Witt C, Beckmann J, Gregorio C, Granzier H, Labeit S. Series of exon-skipping events in titin's elastic spring region as the structural basis for myofibrillar elastic diversity. Circ Res 2000; 86: 1114-1121.

8. Cazorla O, Freiburg A, Helmes M, Centner T, McNabb M, Trombitás K, Labeit S, Granzier H. Differential expression of cardiac titin isoforms and modulation of cellular stiffness. Circ Res 2000; 86: 59-67.

9. Kellermayer MSZ, Smith SB, Bustamante C, GranZIER HL. Complete unfolding of the titin molecule under external force. J Struct Biol 1998; 122: 197205.

10. Helmes M, Trombitas K, Granzier H. Titin develops restoring force in rat cardiac myocytes. Circ Res 1996; 79: 619-626.

11. Price MG. Molecular analysis of intermediate filament cytoskeleton - a putative load-bearing structure. Am J Physiol 1984; 246: H566-H572.

12. Granzier HL, WANG K. Gel electrophoresis of giant proteins: solubilization and silver-staining of titin and nebulin from single muscle fiber segments. Electrophoresis 1993; 14: 56-64.

13. Granzier H, Helmes M, Trombitas K. Nonuniform elasticity of titin in cardiac myocytes: a study using immunoelectron microscopy and cellular mechanics. Biophys J 1996; 70: 430-442.

14. Morano I, Hadicke K, HaAse H, Bohm M, Erdmann E, Schaub MC. Changes in essential myosin light chain isoform expression provide a molecular basis for isometric force regulation in the failing human heart. J Mol Cell Cardiol 1997; 29: 1177-1187.

15. Fitzsimons DP, Moss RL. Role of myosin heavy chain composition in kinetics of force development and relaxation in rat myocardium. J Physiol 1998; 513: 171-183.

16. Metzger JM, Wahr PA, Michele De, Albayya F, WESTFALL MV. Effects of myosin heavy chain isoform switching on $\mathrm{Ca}^{2+}$ activated tension development in single adult cardiac myocytes. Circ Res 1999; 84: 1310-1317.

17. Furst DO, Osborn M, Nave R, Weber K. The organization of titin filaments in the half-sarcomere revealed by monoclonal antibodies in immunoelectron microscopy: a map of ten nonrepetitive epitopes starting at the $\mathrm{Z}$ line extends close to the M line. J Cell Biol 1988; 106: 1563-1572.

18. WANG K, Ramirez-Mitchell R. A network of transverse and longitudinal intermediate filaments is associated with sarcomeres of adult vertebrate skeletal muscle. J Cell Biol 1983; 96: 562-570.

19. Medugorac I. Characterization of intramuscular collagen in the mammalian left ventricle. Basic Res Cardiol 1982; 77: 589-598.

20. Hanley PJ, Young AA, LeGrice IJ, Edgar SG, Loiselle DS. 3-Dimensional configuration of perimysial collagen fibres in rat cardiac muscle at resting and extended sarcomere lengths. I Physiol 1999; 517: 831-837.

21. Kellermayer MS, Smith SB, Granzier HL, Bustamante C. Folding-unfolding transitions in single titin molecules characterized with laser tweezers. Science 1997; 276: 1112-1116.

22. Rodriguez E, Hunter W, Royce M, Leppo M, Douglas A, Weisman H. A method to reconstruct myocardial sarcomere lengths and orientations at transmural sites in beating canine hearts. Am J Physiol 1992; 136: H293-H306.

23. Iwase M, Bishop SP, Uechi M, Vatner DE, Shannon RP, Kudej RK, Wight DC, WAGNER TE, IshikAWA Y, Homcy CJ, Vatner SF. Adverse effects of chronic endogenous sympathetic drive induced by cardiac Gsa overexpression. Circ Res 1996; 78: 517-524.

24. Colbert MC, Hall DG, Kimball TR, Witt SA, Lorenz JN, Kirby ML, Hewett TE, KLevitsky R, Robbins J. Cardiac compartment-specific over-expression of a modified retinoic acid receptor produces dilated cardiomyopathy and congestive heart failure in transgenic mice. J Clin Invest 1997; 100: 19581968.

25. GRIMM AF, Lin HL, GRIMM BR. Left ventricular free wall and intraventricular pressure-sarcomere length distributions. Am J Physiol 1980; 239: H101-H107.

26. MacKenna DA, Omens JH, McCulloch AD, Covell JW. Contributions of collagen matrix to passive left ventricular mechanics in isolated rat hearts. $A m ~ J$ Physiol 1994; 266: H1007-H1018. 